\title{
PMU-based Linear State Estimation of Lausanne Subtransmission Network: Experimental Validation
}

\author{
Lorenzo Zanni*, Asja Derviškadić ${ }^{\dagger}$, Marco Pignati*, Chenxi Xu ${ }^{\ddagger}$, Paolo Romano*, \\ Rachid Cherkaoui $^{\dagger}$, Ali Abur ${ }^{\ddagger}$ and Mario Paolone ${ }^{\dagger}$ \\ * Zaphiro Technologies Sàrl, CH-1015 Lausanne, Switzerland \\ $\dagger$ École Polytechnique Fédérale de Lausanne, CH-1015 Lausanne, Switzerland \\ $\ddagger$ Department of Electrical and Computer Engineering, Northeastern University, Boston, MA 02115 USA
}

\begin{abstract}
The paper presents the implementation details and the experimental validation of a linear state estimator based on synchrophasor measurements in a real subtransmission network. The data are provided by 15 phasor measurement units (PMUs) installed in the 125-kV grid of the city of Lausanne, Switzerland. The PMU-based monitoring infrastructure, the telecommunication and the phasor data concentrator are described in detail. We compare the performance of two different state estimation methods, i.e., the linear weighted least squares and the least absolute value estimator, with a focus on the influence of the line parameter errors on the state estimates. We further analyze the latency contribution associated to each step of the measurement chain, in order to validate its appropriateness to serve timecritical power system applications.
\end{abstract}

Index Terms-Least absolute value, Linear state estimation, Phasor measurement unit, Weigthed least squares.

\section{INTRODUCTION}

In the last decades, state estimation (SE) has become a core power system situation awareness functionality for network operators. Typically, power system state estimators consist in a non-linear weighted least squares (WLS) process, that computes the most likelihood system-state through the statistical processing of real and pseudo-measurements. The accuracy and reliability of the state estimates are exploited by various functionalities, like voltage control, contingency and stability analysis, security assessment, and protections tuning.

One of the main features of SE is the capability to detect, identify, and eliminate large measurement errors, also called bad-data. Most state estimators (e.g., the WLS) need to be coupled with a bad-data processing method, such as the wellknown largest normalized residual (LNR) method [1]. By contrast, the so-called robust estimators, e.g., the least absolute value (LAV), possess an intrinsic bad-data rejection property, thus there is no need to employ a separate bad-data processor [2]. Also, the vulnerability to leverage measurements can be eliminated by strategic scaling without affecting the state estimates, as demonstrated in [2]. In terms of computational time, LAV becomes competitive with WLS, in particular in presence of bad data.

This work was supported by the Swiss Federal Office of Energy (OFEN) under Grant SI/501080-01. The Authors would like to thank Services Industriels de Lausanne (SiL) for the collaboration.
The performance of the aforementioned estimators can be improved if the measurements adopted to estimate the system state are entirely composed of synchrophasors provided by Phasor Measurement Units (PMUs). Indeed, a state estimator that uses only synchrophasor measurements has the following benefits compared to conventional estimators:

1) The measurements are a linear function of the state variables, which leads to a linear state estimator (LSE) consisting in a non-iterative algorithm with low computational time;

2) The measurements are time-stamped, so that they can be time-aligned at the data collection point. This ensures that every set of measurements given to the LSE is composed of measurements taken at the same instant;

3) As the PMUs directly measure the phase-angle, there is no need to choose a reference bus, at which the voltage phase-angle is fixed to an arbitrarily selected value [3];

4) The very high PMU streaming-rate (tens of frames-persecond (fps)) leads to a state estimator characterized by high refresh-rate and low latency, which is called realtime state estimation (RTSE);

5) PMUs measure by default the current and voltage phasors in each phase separately, so that three-phase SE can be used irrespectively of the network-parameter symmetry and power-flow balance.

In the current literature, few papers have described the implementation details and experimental results of LSEs in real power systems using PMUs, e.g., [4], [5], [6]. These previous contributions are limited to the description of the SE method and implementation aspects, lacking a thorough analysis of the SE results. In this paper, we present the SE results obtained using the LAV and the LWLS estimators for the $125-\mathrm{kV}$ subtransmission network of Lausanne city (Switzerland) that is operated by the utility Service Industriels de Lausanne (SiL). The originality of this work lies in a detailed performance assessment of the SE results provided by LSEs that use real measurements taken in a real power network extensively equipped with PMUs. First, we present and discuss the results of the LWLS and LAV estimators. Second, we perform a latency assessment of every element of the chain comprising the PMU, the telecom network, the Phasor Data Concentrator (PDC) and the SE process. 

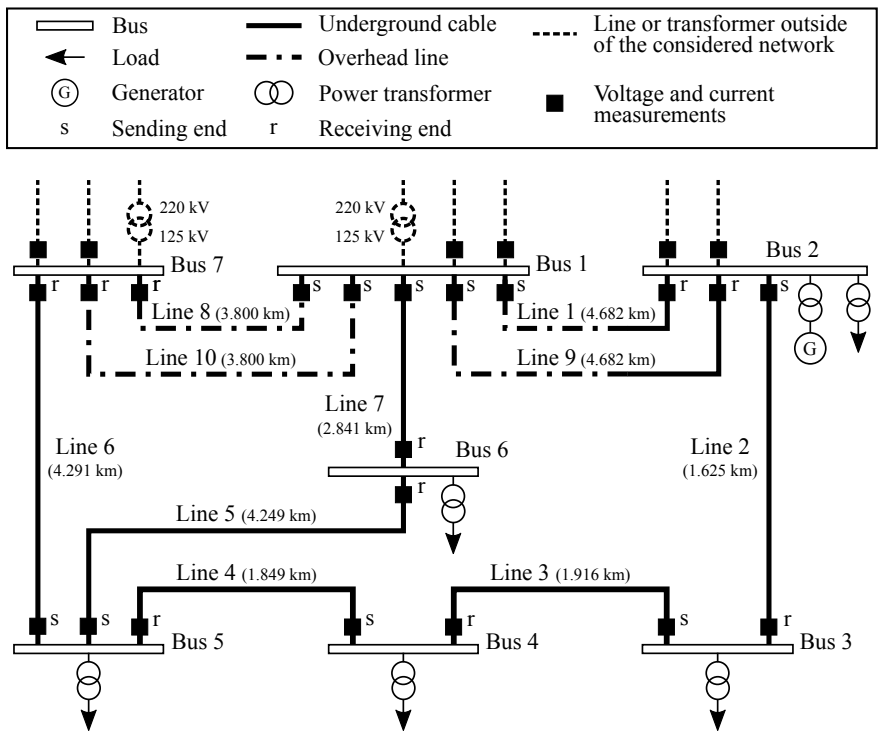

Fig. 1: Network topology of the monitored portion of the 125$\mathrm{kV}$ subtransmission network of Lausanne city, together with the PMU placement. The sending and receiving ends of the lines are denoted by $s$ and $r$, respectively.

The paper is structured as follows. Section II describes the topology of the network and of the PMU placement. Section III provides implementation details of the sensing infrastructure and of the synchrophasor network. Section IV formulates the studied SE methods. Section V assesses the accuracy of the employed SE methods and the time-latency of the overall SE process. Section VI concludes the paper.

\section{Network StRUCtURE AND PMU PLACEMENT}

For the study presented in this paper, we use the measurements provided by PMUs installed in the $125-\mathrm{kV}$ network that supplies the city of Lausanne. Fig. 1 shows the network structure and the PMU placement. The topology is composed of 7 buses and 10 lines. Buses \#1 and \#7 are connected to the higher voltage grid through step-up transformers. No zeroinjection buses are present in this network. The statistics of the power absorbed by the loads are reported in Table I.

We installed 15 PMUs and each PMU can acquire signals from up to two three-phase departing lines by using multiple input-modules. Three-phase nodal-voltage and current-flow synchrophasors are measured at both ends of each line. The full set of measurements is therefore composed of 20 threephase voltage phasors and 20 three-phase current-flow phasors. Note that multiple voltage measurements are available at each bus. Overall, we estimate 42 state variables by using 240 measurements. The pervasive PMU deployment leads to a high measurement redundancy equal to 5.7, which enhances the SE reliability and benefits. A line breaker is present at both ends of each line and the breaker status is included in the PMU packet, thus enabling the real-time knowledge of the network topology. The network model is built from the network topology and line parameters provided in Table II.
TABLE I: 2015 year statistics of the load absorbed-power.

\begin{tabular}{ccccccc}
\hline & & Bus 2 & Bus 3 & Bus 4 & Bus 5 & Bus 6 \\
\hline$P_{\text {max }}$ & {$[\mathrm{MW}]$} & 55 & 26 & 30 & 30 & 24 \\
$P_{\min }$ & {$[\mathrm{MW}]$} & 12 & 8 & 10 & 10 & 9 \\
$P_{\text {mean }}$ & {$[\mathrm{MW}]$} & 30 & 15 & 18.5 & 18.5 & 15 \\
\hline
\end{tabular}

TABLE II: Line parameters: zero (0) and positive (1) sequence.

\begin{tabular}{llllcllc}
\hline & $L$ & $R_{0}$ & $X_{0}$ & $B_{0}$ & $R_{1}$ & $X_{1}$ & $B_{1}$ \\
& {$[\mathrm{~km}]$} & {$[\Omega / \mathrm{km}]$} & {$[\Omega / \mathrm{km}]$} & {$[\mathrm{S} / \mathrm{km}]$} & {$[\Omega / \mathrm{km}]$} & {$[\Omega / \mathrm{km}]$} & {$[\mathrm{S} / \mathrm{km}]$} \\
\hline Line 1 & 4.682 & 0.217 & 0.756 & $14.3 \mathrm{e}-6$ & 0.112 & 0.372 & $15.1 \mathrm{e}-6$ \\
Line 2 & 1.625 & 0.168 & 0.093 & $63.2 \mathrm{e}-6$ & 0.051 & 0.205 & $63.2 \mathrm{e}-6$ \\
Line 3 & 1.916 & 0.168 & 0.093 & $63.2 \mathrm{e}-6$ & 0.051 & 0.205 & $63.2 \mathrm{e}-6$ \\
Line 4 & 1.849 & 0.185 & 0.102 & $60.4 \mathrm{e}-6$ & 0.051 & 0.210 & $60.4 \mathrm{e}-6$ \\
Line 5 & 4.249 & 0.177 & 0.498 & $57.2 \mathrm{e}-6$ & 0.061 & 0.201 & $57.2 \mathrm{e}-6$ \\
Line 6 & 4.291 & 0.168 & 0.093 & $63.2 \mathrm{e}-6$ & 0.051 & 0.205 & $63.2 \mathrm{e}-6$ \\
Line 7 & 2.841 & 0.226 & 0.611 & $57.4 \mathrm{e}-6$ & 0.064 & 0.210 & $57.4 \mathrm{e}-6$ \\
Line 8 & 3.800 & 0.420 & 1.272 & $1.8 \mathrm{e}-6$ & 0.159 & 0.410 & $2.8 \mathrm{e}-6$ \\
Line 9 & 4.682 & 0.217 & 0.756 & $14.3 \mathrm{e}-6$ & 0.112 & 0.372 & $15.1 \mathrm{e}-6$ \\
Line 10 & 3.800 & 0.420 & 1.272 & $1.8 \mathrm{e}-6$ & 0.159 & 0.410 & $2.8 \mathrm{e}-6$ \\
\hline
\end{tabular}

\section{SYSTEM ARCHITECTURE}

This section describes the measurement infrastructure that comprises transducers, PMUs, telecommunication network and Phasor Data Concentrator (PDC).

\section{A. PMU-based monitoring infrastructure}

The monitoring infrastructure is composed of 15 PMUs that receive the voltage and current signals from conventional potential (PTs) and current transformers (CTs). The PT and CT accuracy-classes are specified in Table III, and the PT and CT full-scales are $125 \mathrm{kV}$ and $600 \mathrm{~A}$, respectively. The ratio errors and phase displacements are derived from the accuracy class as indicated in the IEC Std. 61869 [7], [8].

The PMUs are based on the National Instruments (NI) Grid and Automation System [9], a programmable and customizable intelligent electronic device (IED) with PMU capabilities that was designed and tested for standard-compliant electrical substation installations. The absolute time-synchronization is given by a stationary GPS-module that provides the UTCtime information with a maximum uncertainty of $100 \mathrm{~ns}$. The system is also equipped with an 8-channels digital-input module used to monitor in real-time the status of the breakers.

TABLE III: Accuracy class of PTs and CTs.

\begin{tabular}{ccccc}
\hline$\#$ & PT sending & CT sending & PT receiving & CT receiving \\
\hline Line 1 & 0.2 & 0.2 & 0.2 & 0.2 \\
Line 2 & 0.2 & 0.5 & 0.5 & 0.5 \\
Line 3 & 0.5 & 0.5 & 0.2 & 0.5 \\
Line 4 & 0.2 & 0.5 & 0.2 & 0.5 \\
Line 5 & 0.2 & 0.2 & 0.5 & 0.5 \\
Line 6 & 0.2 & 0.5 & 0.2 & 0.5 \\
Line 7 & 0.2 & 0.2 & 0.5 & 0.5 \\
Line 8 & 0.2 & 0.2 & 0.2 & 0.2 \\
Line 9 & 0.2 & 0.2 & 0.2 & 0.2 \\
Line 10 & 0.2 & 0.2 & 0.2 & 0.2 \\
\hline
\end{tabular}



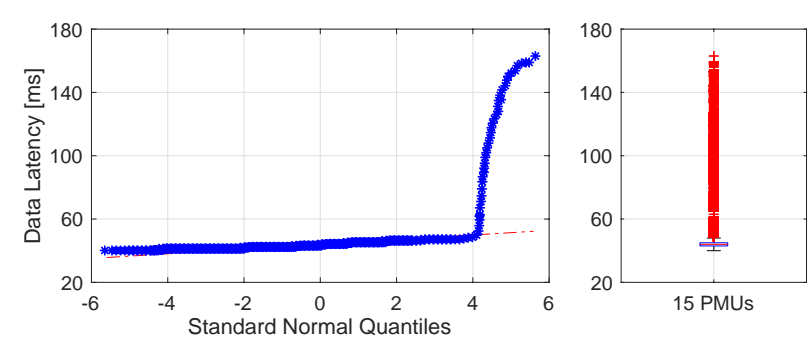

Fig. 2: qq-plot (left) and box-plot (right) representation of the telecom network latency during 24-hours (Sept 7, 2016).

The PMU implements the synchrophasor estimation algorithm presented in [10] and has been certified to be compliant with every class-P requirements and most of the class- $\mathrm{M}$ ones, with the exception of the out-of-band interference test. The PMU is characterized by a maximum total vector error (TVE) of $0.02 \%$ that is maintained in both steady-state and most of the dynamic conditions, irrespectively of the harmonicdistortion level. The PMUs stream the synchrophasors with a reporting rate of $50 \mathrm{fps}$, and are characterized by a mean PMU measurement reporting latency of $44 \mathrm{~ms}$ and standard deviation of $0.3 \mathrm{~ms}$ [11]. Note that the transducer errors are largely predominant compared to the TVE value of PMUs; therefore, the measurement uncertainties used by the SE processes account only for the transducer errors.

\section{B. Telecommunication network}

PMU stream data through the legacy optical-fiber of SiL: each PMU is connected to a switch through an Ethernet cable. The communication is established through a dedicated virtual LAN. Optical-fiber represents the favourite communication layer to transmit data at the high streaming-rate of PMUs, as it guarantees fast and reliable data delivery and enables a large data traffic. In this respect, Fig. 2 shows the aggregated endto-end latency of PMU data measured over 24-hours across all PMU data streams, on September 7th, 2016. As it can be noticed, the data delivery is quite deterministic and is characterized by very few outliers. The majority of the values is concentrated around the median of $44 \mathrm{~ms}$, and outliers are upper bounded by the maximum value of $163 \mathrm{~ms}$.

\section{Phasor data concentrator}

The adopted PDC architecture is presented in [11], and implements the so-called absolute time data pushing logic, therefore the time-aligned datasets are reported at a constant reporting rate (corresponding to the PMU one, i.e., $50 \mathrm{fps}$ ). To do so, the PDC is synchronized to an absolute time reference, provided by a GPS card characterized by an accuracy of 100 ns. By using such logic, the latency variations in receiving the data frames, introduced by the underlying telecommunication media, are completely compensated by the PDC wait time, resulting in a periodic reporting of time-aligned data-sets to the SE process. According to the results presented in Fig. 2, the absolute PDC wait time is set to $60 \mathrm{~ms}$ : such value allows to collect a consistent number of outliers without scarifying the timing needs of overlying time-critical applications.

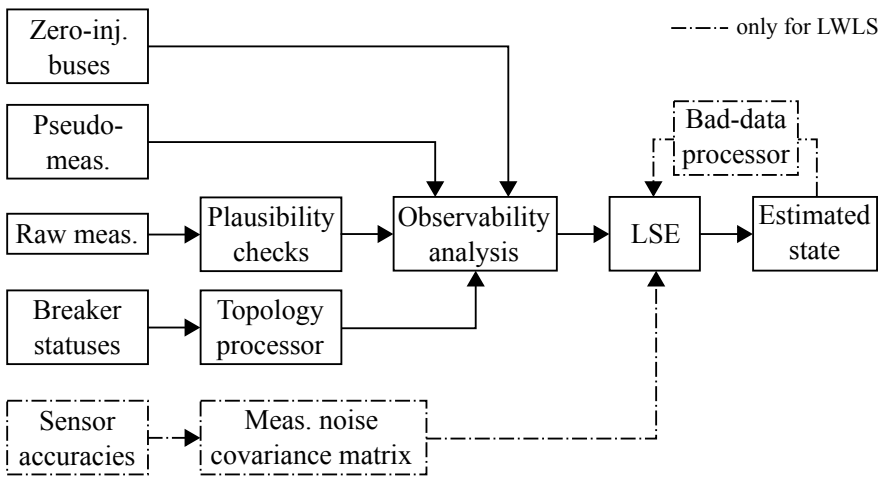

Fig. 3: Flow diagram of the LSE process.

The PDC is placed in the control room of SiL, located by bus \# 2 (see Fig. 1), and is integrated in a workstation equipped with an Intel Xeon Processor at $2.4 \mathrm{GHz}, 8 \mathrm{~GB}$ of RAM and running Windows Server 2008.

\section{STATE ESTIMATION METHODS AND IMPLEMENTATION}

The SE process is composed of a series of functions depicted in the flow diagram of Fig. 3.

The raw measurements undergo plausibility checks in order to identify gross errors. The topology processor builds the network topology based on the status of the breakers ${ }^{1}$. Then, an observability analysis is performed in order to check whether the entire grid is observable given the topology and the available measurements (comprising real-time measurements as well as possible pseudo-measurements and zero-injectionbuses virtual-measurements). If not, the grid can be split in observable islands and a separate SE is performed for every island. The network model is built with the network topology and parameters. The LWLS needs also the measurement noise covariance matrix that represents the uncertainties of the measurements. Finally, the LSE computes the estimated state. Afterwards, the LWLS uses a bad-data processor to identify and eliminate possible bad-measurements; after the elimination procedure, the estimated state is recomputed and this process is iterated until no bad measurements are detected.

\section{A. Plausibility checks, topology processor and observability}

A first plausibility check consists in analyzing the SYNCH, STAT and CHK fields (see [12]) in order to detect and remove measurements coming from PMUs that (1) have detected a loss of external time synchronization or a time input connection failure, or (2) are providing data frames with corrupted bits.

The status of the breakers streamed by PMUs are used by the topology processor to update in real-time the network topology. In case of missing/removed measurements, the breaker status is set to the last available status or to a userdefined normal status. We have implemented also a topologyerror check that detects an error in the OPEN breaker status: when the active-power flow measurement is higher than 100

\footnotetext{
${ }^{1} \mathrm{~A}$ more robust implementation could integrate a sophisticated plausibility check also of the status of the breakers.
} 
$\mathrm{kW}$ and the breaker status streamed by the PMU is $O P E N$, the breaker status is overwritten as CLOSED. Instead, we are not able to detect an error in the CLOSED breaker status, because it can occur that the breaker status is CLOSED and the power flow is zero, at the same time.

Then, given the topology and the set of measurements, we build the measurement matrix $\mathbf{H}$ that links the state variables to the measurements (see Section IV-B1 for further details). If $\mathbf{H}$ is of full rank, we proceed with the SE; if not, we give a warning and wait for the next time-step. Note that, due to the very high redundancy, the case of unobservable network is not likely to occur.

\section{B. State estimation methods}

We recall here below the formulations and characteristics of the two SE methods employed in this paper.

1) Linear weighted least squares: The LWLS is a static state-estimator that uses only the information contained in the current set of measurements $\mathbf{z} \in \mathbb{R}^{m}$ [1], [13]. The linear measurement model is

$$
\mathbf{z}_{k}=\mathbf{H}_{k} \mathbf{x}_{k}+\mathbf{v}_{k}
$$

where $k$ is the discrete-time index, $\mathbf{x}_{k} \in \mathbb{R}^{n}$ is the system state, $\mathbf{H}_{k}$ is the measurement matrix, and $\mathbf{v}_{k}$ is the measurement noise that is assumed to be a white Gaussian sequence with covariance matrix $\mathbf{R}_{k}$. The network state is composed of the real and imaginary parts of the nodal phase-to-ground voltages at every bus and in each of the three phases. Then, the estimated state $\widehat{\mathbf{x}}_{k}$ is computed as

$$
\widehat{\mathbf{x}}_{k}=\mathbf{G}_{k}^{-1} \mathbf{H}_{k}^{T} \mathbf{R}_{k}^{-1} \mathbf{z}_{k} .
$$

where $\mathbf{G}_{k}$ is the so-called Gain matrix:

$$
\mathbf{G}_{k}=\mathbf{H}_{k}^{T} \mathbf{R}_{k}^{-1} \mathbf{H}_{k} .
$$

2) Least absolute value: The objective of the LAV estimator is to minimize the sum of the absolute values of the measurement residuals $\mathbf{r}_{k}$ as [1], [2]:

$$
\begin{aligned}
\min & \mathbf{c}^{T}\left|\mathbf{r}_{k}\right| \\
\text { subject to: } & \mathbf{z}_{k}-\mathbf{H}_{k} \widehat{\mathbf{x}}_{k}=\mathbf{r}_{k}
\end{aligned}
$$

where $\mathbf{c}$ is a vector of ones. This optimization problem can be re-arranged as an equivalent linear programming problem. Note that the LAV does not require neither the definition of the matrix $\mathbf{R}_{k}$ nor separate bad-data processing. In the ideal case of white Gaussian measurement noise with the exact knowledge of $\mathbf{R}_{k}$, the LWLS remains the optimal estimator. However, such assumptions is not true for real systems.

\section{Measurement-noise covariance matrix}

The measurement-noise covariance matrix $\mathbf{R}$ is assumed to be diagonal, as we neglect the correlation among different measurements for the reasons stated in [13]. The diagonal entries of $\mathbf{R}$ are the variances that take into account the errors of PTs/CTs and PMUs. However, we consider only the PT/CT errors, since the PMU error is more than a order of magnitude smaller than the PT/CT uncertainties [10].
Under these assumptions, the procedure to derive the diagonal elements of the measurement-noise covariance matrix $\mathbf{R}$ is carefully explained in section 4.2.1 of [14], and briefly summarized in what follows. The measurement variances are evaluated from the limits of ratio error and phase displacement defined by the PT and CT accuracy classes (given in the IEC Std. 61869 [7] and [8], respectively). As indicated in the Guide to the expression of uncertainty in measurement (GUM) [15], we assume a Gaussian error distribution ${ }^{2}$. Therefore, we divide these limits by a coverage factor equal to 3 in order to obtain the standard deviations. These limits are defined as a function of the measurand, therefore, they change continuously over time together with the grid operating conditions. It is worth pointing out that, as indicated in [7], [8], as long as the PT/CT loading conditions defined in these standards are respected, the error limits are only a function of the measurand, and not of the loading condition. The final step consists in the projection of the variances from polar to rectangular coordinates as LSE uses measurements in rectangular coordinates [13].

\section{Bad-data processor}

The WLS is vulnerable to bad-data that cause biased estimates; indeed, they require a post-estimation method that detects and identifies bad-data. We have implemented the wellknown largest normalized residual (LNR) test [1]. The latter detects the presence of bad-data if the LNR exceeds a certain threshold $\epsilon$ (usually equal to 3 or 4 ) and flags the related measurement as a bad-data. Then, the SE is re-computed without using this measurement. The procedure is iterated until no bad-data are detected.

On the contrary, the bad-data rejection is an intrinsic property of the LAV, which does not need a separate bad-data processor. The LAV is still vulnerable to leverage measurements, because the residuals of the leverage measurements remain little even in presence of large measurement-errors. This drawback can be eliminated with a proper scaling of $\mathbf{H}$ without affecting the SE result, thanks to the linearity of the measurement model, as explained in detail in [2].

\section{State estimation RESUlts}

In this section, we present and discuss the estimation values obtained with the following state estimators:

- LWLS estimator without bad-data processing;

- LWLS estimator that uses the LNR test to identify and remove bad data, called $L W L S$ - LNR (the threshold of the LNR-test is set to 4);

- LAV estimator.

In addition, we present a latency assessment of each element of the process, from the PMUs to the SE output.

\section{A. Accuracy assessment}

We consider the network in quasi-steady state conditions when the state changes smoothly and no particular events (e.g., line switching or faults) take place. The analyses carried out

\footnotetext{
${ }^{2}$ A more realistic PT and CT error model could be estimated online [16].
} 
TABLE IV: Three-phase power-flow measurements at a specific time-step (the first of the considered time-window), at both ends of every line (sending $s$ and receiving $r$ ).

\begin{tabular}{lcccccc}
\hline & $P s_{a}$ & $P s_{b}$ & $P s_{c}$ & $Q s_{a}$ & $Q s_{b}$ & $Q s_{c}$ \\
{$[\mathrm{MW}]$} & {$[\mathrm{MW}]$} & {$[\mathrm{MW}]$} & {$[\mathrm{MVAR}]$} & {$[\mathrm{MVAR}]$} & {$[\mathrm{MVAR}]$} \\
\hline Line 1 & 10.896 & 10.673 & 10.825 & -0.062 & -0.738 & -0.686 \\
Line 2 & 2.864 & 1.977 & 2.122 & 0.402 & 0.491 & -0.562 \\
Line 3 & -3.701 & -4.366 & -4.360 & -0.511 & -0.473 & -1.236 \\
Line 4 & -12.807 & -13.103 & -13.258 & -2.241 & -2.305 & -2.701 \\
Line 5 & -6.055 & -6.127 & -6.380 & -0.069 & 0.047 & -0.126 \\
Line 6 & -14.502 & -14.428 & -14.538 & -1.584 & -1.825 & -1.684 \\
Line 7 & 11.559 & 11.370 & 11.693 & -0.950 & -0.917 & -1.007 \\
Line 8 & -0.364 & -0.073 & -0.265 & -0.893 & -0.939 & -0.634 \\
Line 9 & 10.769 & 10.588 & 11.095 & -0.483 & -0.342 & -0.421 \\
Line 10 & -0.375 & -0.065 & -0.262 & -0.893 & -0.921 & -0.651 \\
& & & & & & \\
& $P r_{a}$ & $P r_{b}$ & $P r_{c}$ & $Q r_{a}$ & $Q r_{b}$ & $Q r_{c}$ \\
& {$[\mathrm{MW}]$} & {$[\mathrm{MW}]$} & {$[\mathrm{MW}]$} & {$[\mathrm{MVAR}]$} & {$[\mathrm{MVAR}]$} & {$[\mathrm{MVAR}]$} \\
\hline Line 1 & -10.872 & -10.652 & -10.808 & -0.301 & 0.366 & 0.312 \\
Line 2 & -2.841 & -1.956 & -2.122 & -0.908 & -1.002 & 0.056 \\
Line 3 & 3.708 & 4.371 & 4.386 & -0.129 & -0.170 & 0.612 \\
Line 4 & 12.801 & 13.109 & 13.255 & 1.698 & 1.784 & 2.172 \\
Line 5 & 6.052 & 6.137 & 6.384 & -1.152 & -1.272 & -1.107 \\
Line 6 & 14.519 & 14.448 & 14.562 & 0.214 & 0.452 & 0.312 \\
Line 7 & -11.572 & -11.375 & -11.704 & 0.072 & 0.057 & 0.139 \\
Line 8 & 0.354 & 0.074 & 0.265 & 0.827 & 0.880 & 0.573 \\
Line 9 & -10.752 & -10.563 & -11.080 & 0.118 & -0.018 & 0.048 \\
Line 10 & 0.376 & 0.082 & 0.261 & 0.859 & 0.883 & 0.598 \\
\hline
\end{tabular}

in this section refer to a 200 seconds time-window on August 16th 2016 at 8 a.m. UTC time (10 a.m. Swiss local time).

A snapshot of the grid operating-conditions is shown in Table IV, which contains the power measurements at both ends of each line (derived from the voltage and current synchrophasors) at the first time-step of the considered timewindow. The sending and receiving line-ends are specified in Fig. 1 and the power-flow is positive if exiting from the bus. Note the presence of not negligible active-power flow imbalances among the phases of a line (e.g., almost $1 \mathrm{MW}$ difference between phases $a$ and $b$ of line \#2) and between the two parallel lines \#1 and \#9 (about $300 \mathrm{~kW}$ in phase $c$ ). Similar observations can be made for the reactive power; therefore, a three-phase state estimator is particularly useful in this case.

The voltage magnitude and phase-angle measurements are plotted in Fig. 4 together with the estimates provided by the LWLS estimator. The voltage estimates of the other two state estimators (LWLS-LNR and LAV) are not shown in this figure, as they are very similar to those of the LWLS. It can be seen that the errors introduced by transducers are compensated by the state estimators that provide a consistent profile of the estimated voltages. In particular, the measured voltage magnitude at bus \#6 is affected by a visible bias close to the limit of the PT accuracy class. Also, all voltage magnitudes and phase-angles are close to each other, because the grid is composed of electrically short and not heavily loaded lines.

To give an idea of the time-evolution of the voltage, Fig. 5 shows the voltage measurements and estimates at bus \#1 with a reporting time of $20 \mathrm{~ms}$ and an observation interval of $30 \mathrm{~s}$. As shown in Fig. 1, bus \#1 is equipped with 5 PMUs, therefore
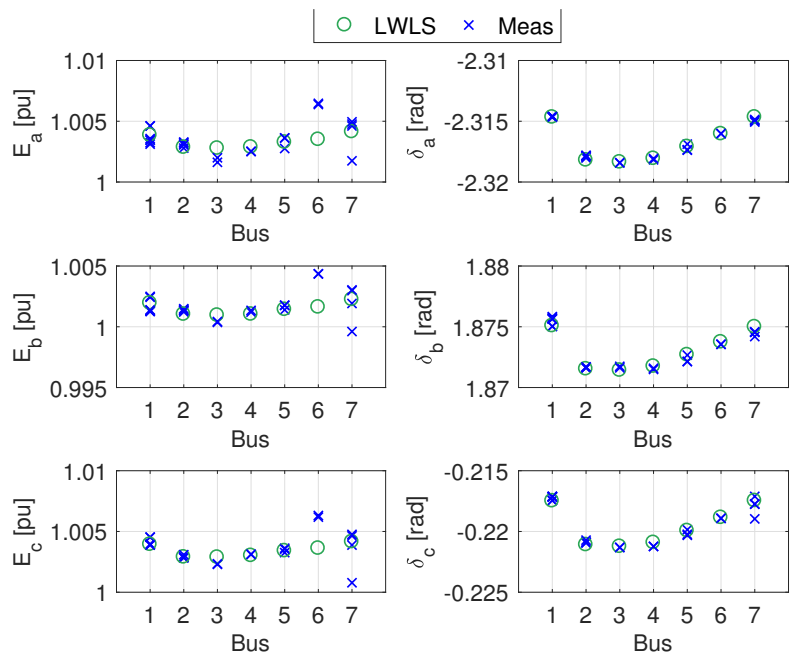

Fig. 4: Voltage magnitude and phase at every bus at a specific time-step $k=1$.

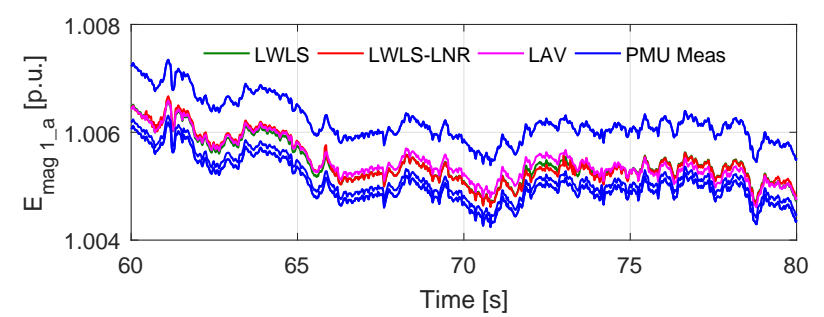

Fig. 5: Time evolution of the measured and estimated voltage magnitude at bus \#1.

5 measurements are provided (some plots are superimposed). Here the voltage estimates of the three state estimators are almost superimposed.

On the contrary, the time evolution of the current estimates shown in Fig. 6 shows a very different behavior of the state estimators. The LAV estimates oscillate between the measurement values and the LWLS estimates, whereas the LWLS-LNR estimates have even larger and sudden changes. Indeed, 18 measurements on average are identified as bad-data at every time-step by the LNR-test. The normalized residuals of voltage measurements are lower than 2 , whereas the large majority of the normalized residuals of current measurements are higher than the threshold set for the LNR-test (equal to 4), as we can observe in Fig. 7 (only real parts of the sending-end current-flow measurements are shown as imaginary parts and receiving-end have similar residuals). The question is whether all measurements flagged as bad-data contain real gross errors or the LNR-test is giving erroneous results.

To confirm this hypothesis, an off-line simulation to assess the impact of line-parameter errors in this specific grid is carried out. Using a power flow solution corresponding to the loading of the real grid, 10000 state estimations are executed where different combinations of random parameter errors in the $\pm 5 \%$ range are added to all transmission lines. Fig. 8 shows the statistics of the normalized residuals obtained 


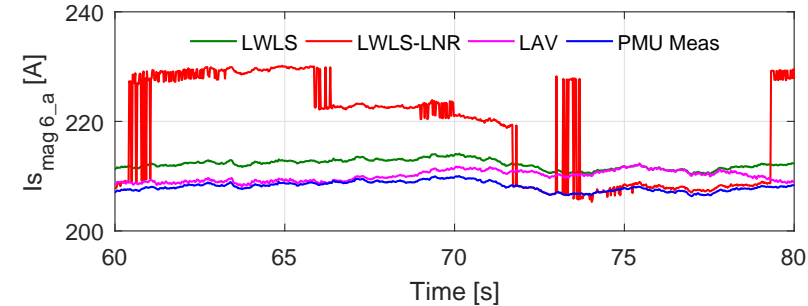

Fig. 6: Time evolution of the measured and estimated current magnitude at line \#6.

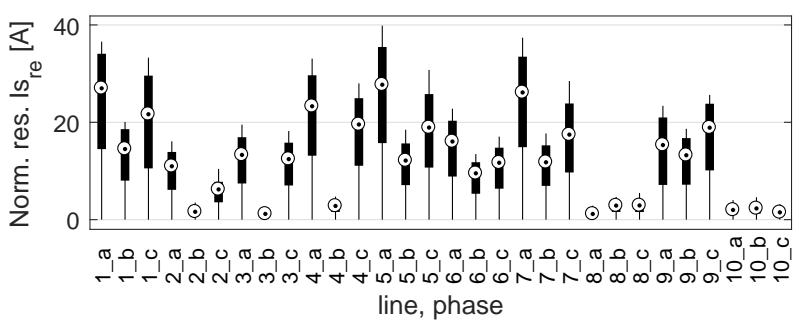

Fig. 7: Statistics of the distributions of the normalized residuals related to the real-part of the sending-end current measurements of the LWLS estimator (similar residuals are obtained for the imaginary parts and for the receiving-end currents).

with this procedure. We can observe that relatively small errors in estimating line parameters (smaller than 5\%) produce normalized residuals in the range of the ones observed in the real grid. Therefore, since it is plausible that $\pm 5 \%$ errors are present in the parameters, we can conclude that the high normalized residuals are due to line-parameter errors and not to gross errors in the measurements. In this case, the LNR-test erroneously flags some measurements as bad-data producing the undesired behavior observed in Fig. 6.

After having clarified the cause of the small deviations of the estimated values from the measured values, we can compare the residuals (difference between estimated and measured quantity, expressed in Amperes) related to the sendingend current-flow magnitudes for the three state estimators, which are displayed in Fig. 9. As expected, the LWLS-LNR exhibits the highest residuals in some time-steps, because good measurements are erroneously eliminated. The LWLS and

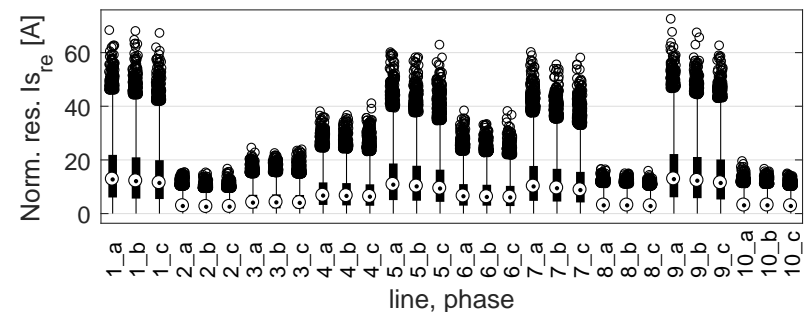

Fig. 8: Off-line simulation where random errors are added to the line parameters in the range $\pm 5 \%$. Normalized residuals related to the real-part of the sending-end current measurements of the LWLS estimator (similar residuals are obtained for the imaginary parts and for the receiving-end currents).

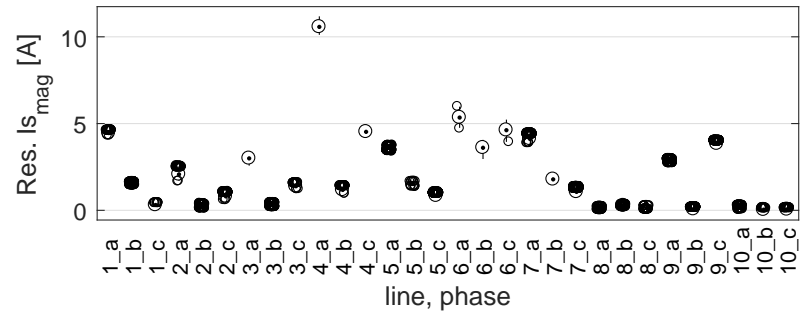

(a) LWLS

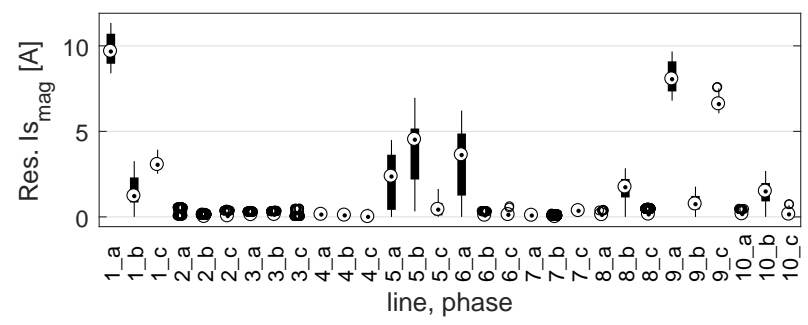

(b) LAV.

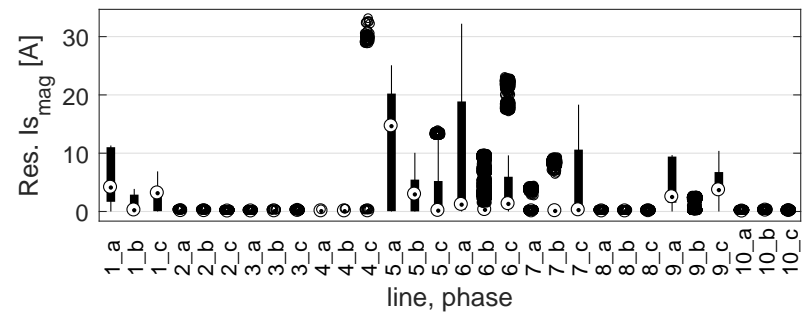

(c) LWLS-LNR

Fig. 9: Statistics of the distributions of the residuals of the real parts of the sending-end current-flow measurements (similar residuals are obtained for the imaginary parts and for the receiving-end currents).

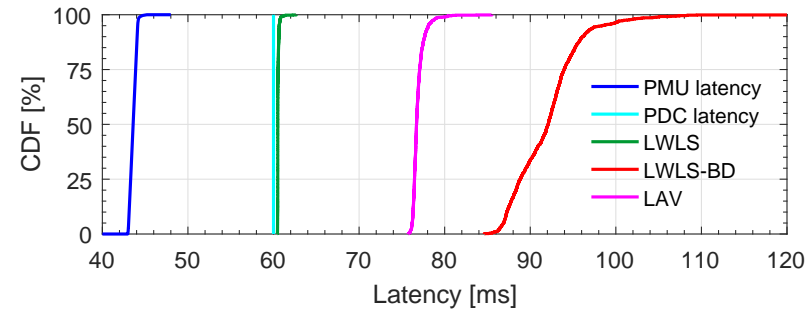

Fig. 10: Time-latency assessment: CDF of the time latency associated with the analyzed SE processes.

LAV residuals are smaller than 10 A along the 200 seconds time-window, which are quite small (less than 6\%) compared to the measured current values. Therefore, the LWLS and LAV estimates can be considered to be accurate enough and the baddata identification threshold could be increased from 4 to 50 or 100 in order to avoid flagging good measurements as baddata due to errors in the line parameters. In this case, since no measurements are eliminated by the LNR test, the LWLS-LNR and the LWLS estimators use the same set of measurements and their results will be identical. 


\section{B. Time-latency assessment}

This section is meant to assess the timing performance of the overall SE process in order to demonstrate the appropriateness of the developed tools to serve real-time power system applications. The total time-latency of the process is defined as the time interval between the central instant of the PMU acquisition time-window and the instant the SE provides the estimated state. To this end, the PDC is equipped with a GPS receiver providing the absolute time information with a resolution of $1 \mathrm{~ms}$ and the PMU data flow is tracked along the entire chain of events, measuring:

- The data frame arrival time in the PDC (PMU latency);

- The time-aligned dataset push time (PDC latency);

- The SE computation time.

Figure 10 shows the cumulative distribution function (CDF) of the latency of every step of the above-described chain, considering the same data samples used in Section V-A. As evident from the figure, the PDC absolute time data pushing logic enables the cancellation of the latency variations introduced by the telecommunication network: even though data are collected by the PDC between 40 and $48 \mathrm{~ms}$, timealigned datasets are streamed to the SE with a latency that is exactly $60 \mathrm{~ms}$ for every data frame.

Depending on the computational complexity of the adopted SE method, the final latency can vary widely. As expected, the LWLS is the fastest algorithm, characterized by $0.5 \mathrm{~ms}$ mean latency, as it accounts for the lower computational burden. By contrast, the LWLS-LNR is the slower one (31 ms on average), as, at each time-step, it has to compute the covariance matrix of the residuals and re-estimate the state as many times as the number of identified bad-data. Indeed, in the considered observation interval, the LWLS-LNR rejects on average 18 current measurements per time-stamp. Independent of the adopted SE process, the overall computation time is below $120 \mathrm{~ms}$, which is compatible with real-time applications.

\section{CONCLUSION}

The paper described the practical implementation and results of state estimation in the $125-\mathrm{kV}$ subtransmission network of the city of Lausanne, Switzerland. We assessed the SE accuracy using two methods, the LWLS and the LAV, and we measured the time-latency of every element of the process.

We observed that the effect of line parameter errors and measurement errors can be similar, as they can both generate large measurement residuals. This phenomenon causes the LNR-test to erroneously eliminate some measurements from the data set. We showed with offline simulations that even $5 \%$ errors in the line parameters may generate large residuals. In a real grid where both parameter and measurement errors are inevitably present, it is not trivial to discriminate between the two. In the case presented in this paper, thanks to the high measurement redundancy, we could verify that the large residuals are most probably due to line parameter errors. A network operator should first carefully verify the reliability of line parameters in order to ensure a high SE accuracy [17].
Moreover, if the LWLS is used, the bad-data identification threshold used by the LNR-test should be adjusted in order to avoid flagging good measurement as bad-data. The LAV does not suffer from this problem, but has a higher computational time.

The latency assessment has proved that the SE output is made available with very low latency, below $100 \mathrm{~ms}$, which is compatible with time-critical power system applications. The fastest estimator is the LWLS without bad-data processing (overall latency of about $60 \mathrm{~ms}$ ). However, the more bad-data are identified by the LNR-test, the more re-computations of the estimates are required; indeed, we showed that the overall computation time of the LWLS can become higher than that of the LAV.

\section{REFERENCES}

[1] A. Abur and A. G. Exposito, Power system state estimation: theory and implementation. New York City, NY: Marcel Dekker, 2004.

[2] M. Göl and A. Abur, "LAV based robust state estimation for systems measured by PMUs," IEEE Transactions on Smart Grid, vol. 5, no. 4, pp. 1808-1814, July 2014.

[3] A. Abur, "Impact of phasor measurements on state estimation," in Electrical and Electronics Engineering, 2009. ELECO 2009. International Conference on, Nov 2009, pp. I-3-I-7.

[4] K. D. Jones, J. S. Thorp, and R. M. Gardner, "Three-phase linear state estimation using phasor measurements," in IEEE Power and Energy Society General Meeting (PES), 2013.

[5] L. Zhang, A. Bose, A. Jampala, V. Madani, and J. Giri, "Design, testing, and implementation of a linear state estimator in a real power system," IEEE Transactions on Smart Grid, vol. 8, no. 4, pp. 1782-1789, July 2017.

[6] M. Pignati et al., "Real-time state estimation of the EPFL-campus medium-voltage grid by using PMUs," in 2015 IEEE Power Energy Society Innovative Smart Grid Technologies Conference (ISGT), Feb. 2015, pp. 1-5.

[7] "Instrument transformers part 3: Additional requirements for inductive voltage transformers," IEC Std 61869-3, 2011.

[8] "Instrument transformers part 2: Additional requirements for current transformers," IEC Std 61869-2, 2014.

[9] "National Instrument Grid Automation System," http://www.ni.com/white-paper/52556/en/, accessed: 2016-09-08.

[10] P. Romano and M. Paolone, "Enhanced interpolated-DFT for synchrophasor estimation in FPGAs: Theory, implementation, and validation of a PMU prototype," IEEE Transactions on Instrumentation and Measurement, vol. 63, no. 12, pp. 2824-2836, Dec. 2014.

[11] A. Derviškadić, P. Romano, M. Pignati, and M. Paolone, "Architecture and experimental validation of a low-latency phasor data concentrator," IEEE Transactions on Smart Grid, vol. 9, no. 4, pp. 2885-2893, July 2018.

[12] "IEEE standard for synchrophasor data transfer for power systems," IEEE Std C37.118.2-2011 (Revision of IEEE Std C37.118-2005), pp. 1-53, Dec. 2011.

[13] M. Paolone, J.-Y. L. Boudec, S. Sarri, and L. Zanni, Advances in Power System Modelling, Control and Stability Analysis. F. Milano, Ed. Edison, NJ, USA: IET, 2015, ch. 6: Static and recursive PMU-based state estimation processes for transmission and distribution power grids.

[14] L. Zanni, "Power-system state estimation based on PMUs static and dynamic approaches - From theory to real implementation," $\mathrm{PhD}$ Thesis, EPFL, Lausanne, 2017. [Online]. Available: http://infoscience.epfl.ch/record/228451

[15] Joint Committee for Guides in Metrology (JCGM), "Guide to the expression of uncertainty in measurement (GUM)," JCGM 100:2008, 2008.

[16] A. Pal, P. Chatterjee, J. S. Thorp, and V. A. Centeno, "Online calibration of voltage transformers using synchrophasor measurements," IEEE Transactions on Power Delivery, vol. 31, no. 1, pp. 370-380, Feb. 2016.

[17] Jun Zhu and A. Abur, "Identification of network parameter errors," IEEE Transactions on Power Systems, vol. 21, no. 2, pp. 586-592, May 2006. 